\title{
EFFECT OF HYALURONIC ACID ON FRICTION OF ARTICULAR CARTILAGE
}

\author{
D. Rebenda*, P. Čípek, D. Nečas, M. Vrbka, M. Hartl
}

\begin{abstract}
Intra-articular injections of hyaluronic acid based solutions are gaining popularity as a treatment option for non-operative management of patients with degenerative joint diseases. Although there is a plenty of evidence for lowering of friction in large human joints by exogenous hyaluronic acid, the physical phenomenon standing behind these results has not been clarified yet. This leads to large dispersion in effectiveness and duration in action of viscosupplementation as a treatment for osteoarthritis and; subsequently, to disparity between the view of practitioners and recent procedures questioning or disregarding the efficiency of hyaluronic acid injections. In this study, the changes in friction between intact porcine cartilage and glass plate during reciprocating sliding tests were observed to better understand interaction between hyaluronic acid and synovial fluid components. Contact was lubricated with protein solutions both with and without addition of hyaluronic acid. The effectiveness of proteins and influence of hyaluronic acid on friction are discussed.
\end{abstract}

Keywords: articular cartilage, synovial fluid, friction, hyaluronic acid, viscosupplemenatation

\section{Introduction}

Articular cartilage covers sliding surfaces in large synovial joints like hip or knee. It is biological tissue composed of solid and fluid phase, which determines its mechanical properties. Solid phase is composed of extracellular matrix from collagen fibers and proteoglycans. Gaps between matrix are filled with fluid phase consisted of water and electrolytes. It is widely known that articular cartilage maintains great lubricating properties with extremely low friction and minimal wear and can absorb impact loads quite well. These excellent properties are achieved by interaction between its solid and fluid phase (Lees, 2016).

Osteoarthritis is degenerative joint disease characterized by imbalance between synthesis and wear of articular cartilage. It is one of the most common disorders of musculoskeletal system, which affect nearly $70 \%$ of 70 year olds (Forsey, 2006). Surface of cartilage shows arear of softening, fibrillations or erosions. In the later stage, there may even be areas of cartilage loss. One of the noninvasive methods for curing this disease is viscosupplementation, which consist in intra-articular injections with preparations based on hyaluronic acid. The original idea of viscosupplementation (Balazs, 1993) was to improve rheological properties of synovial fluid. Better viscoelasticity of synovial fluid should lead to lower friction and better shock absorption abilities. However, medical studies (Ghosh, 2002) shown positive effect of viscosupplementation even 6 months after injection whilst exogenous hyaluronic acid can be detected in synovial fluid just few weeks after injection. This points out on another effects of hyaluronic acid like synthesis of endogenous hyaluronic acid or anti-inflammatory effect (Altman, 2015). However, the dominant effect of viscosupplementation and physiological phenomenon responsible for these results was not clarified yet. This leads to big dispersion in effectiveness and duration of action.

The effectiveness of hyaluronic acid in lowering friction was already proved. Addition of hyaluronic acid to basic solution - PBS (Bell, 2006) or Ringers solution (Forsey, 2006) leads to decrease of friction coefficient. Interaction between hyaluronic acid and other synovial fluid components seems to be very 
important. Addition of phospholipids to hyaluronic acid solution leads to more pronounced lowering of friction. On the other hand, Schmidt (2007) published data where addition of phospholipids into solution of hyaluronic acid and proteoglycans did not lead to any significant changes in friction coefficient.

Proteins play significant role in lubrication of articular cartilage too. According to Murakami (2007) addition of albumin to basic solution leads to increase of friction in artificial cartilage while addition of $\gamma$-globulin with same concentration leads to decrease of friction. Solution of both proteins reported even lower friction than $\gamma$-globulin. In the following paper (Murakami, 2013) authors mixed protein solutions with hyaluronic acid and phospholipids with different impacts on friction. However, the lowest values of friction coefficient were obtained for solution consisted of all 4 substances.

\section{Materials and Methods}

Reciprocating tests for the sliding pair of stationary flat plate from optical glass (B270) and moving porcine cartilage specimen were conducted in commercial tribometer Bruker UMT TriboLab in pin-on-plate configuration. Coefficient of friction was investigated as a function of time, while the contact was lubricated with various lubricants composed of proteins and hyaluronic acid. Schematic illustration of experimental device is displayed in Fig. 1.

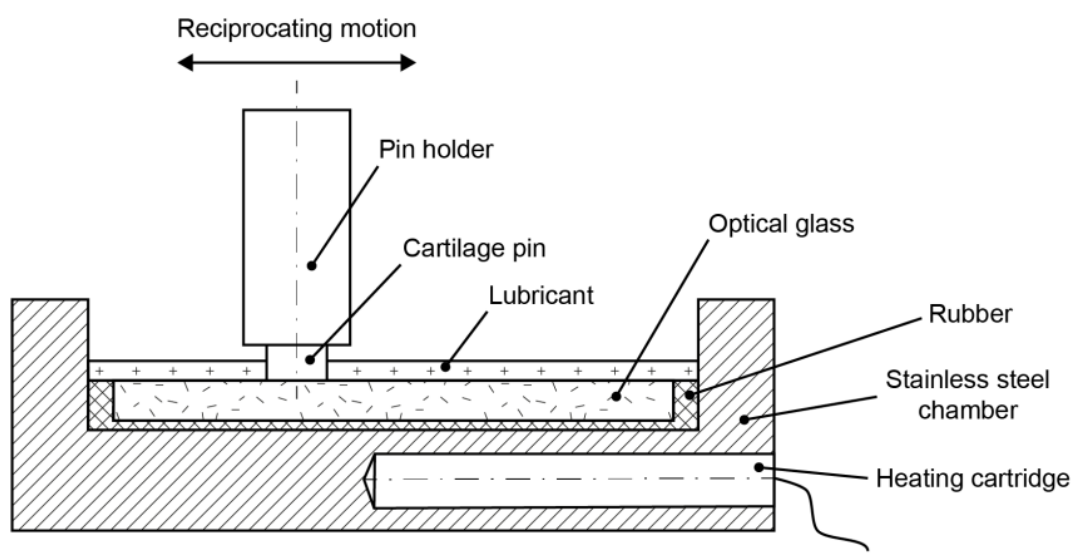

Fig. 1: Scheme of the experimental apparatus.

Intact cartilage specimens with underlying subchondral layer were prepared from porcine femur. Cylindrical cartilage specimens with diameter of $5.6 \mathrm{~mm}$ were extracted from femoral head using a hollow drill. After preparation, the specimens were stored in freezer at $-20^{\circ} \mathrm{C}$ in PBS solution for no more than 2 weeks. It has been reported (Szarko, 2010) that storing articular cartilage under these condition does not change its mechanical properties. Half an hour before the experiment, cartilage was removed from freezer to thaw at room temperature.

Tab. 1: Composition of the test lubricants.

\begin{tabular}{ccccc}
\hline Test fluid & $\begin{array}{c}\text { Albumin } \\
(\mathbf{m g} / \mathbf{m l})\end{array}$ & $\begin{array}{c}\boldsymbol{\gamma} \text {-globulin } \\
(\mathbf{m g} / \mathbf{m l})\end{array}$ & $\begin{array}{c}\text { Hyaluronic acid } \\
(\mathbf{m g} / \mathbf{m l})\end{array}$ & $\begin{array}{c}\text { Volume } \\
(\mathbf{m l})\end{array}$ \\
\hline Albumin & 24.9 & - & - & 6 \\
\hline Albumin + HA & 24.9 & - & 10 & 6 \\
\hline$\gamma$-globulin & - & 6.1 & - & 6 \\
\hline$\gamma$-globulin + HA & - & 6.1 & 10 & 6 \\
\hline Albumin $+\gamma$-globulin & 24.9 & 6.1 & - & 6 \\
\hline Albumin $+\gamma$-globulin + HA & 24.9 & 6.1 & 10 & 6 \\
\hline
\end{tabular}

Lubricants are PBS solutions containing bovine serum albumin, $\gamma$-globulin from bovine blood and hyaluronic acid. The combinations of lubricant constituents used in friction tests and their concentrations are shown in Tab. 1. Concentrations of proteins are based on paper given by Galandáková et al. (2016), where authors performed an extensive study focused on analysis of composition of synovial fluid of different orthopedic patients. Concentrations of albumin and $\gamma$-globulin correspond to synovial fluid of 
patients with end stage of osteoarthritis. Simple protein solutions were prepared and stored overnight in refrigerator to enable proteins to completely dissolve in PBS. Concentration and molecular weight of hyaluronic acid correspond to commercially produced viscosupplements. Hyaluronic acid was prepared in extra solution, and was mixed in 1:1 ratio with protein solution in magnetic stirrer before experiment.

The reciprocating tests were conducted at sliding speed of $10 \mathrm{~mm} / \mathrm{s}$ and stroke length of $20 \mathrm{~mm}$ under constant load of $5 \mathrm{~N}$ resulting to contact pressure equal to $1 \mathrm{MPa}$. To mimic temperature of human body, lubricant was heated to $37^{\circ} \mathrm{C}$ via heating cartridges in steel chamber. Before each experiment, unloaded cartilage sample was immersed in lubricant for $320 \mathrm{~s}$ to let cartilage soak with lubricant. After that, cartilage was loaded and test immediately began. The friction force was continuously monitored through force sensor.

\section{Results and discussion}

First set of experiments was conducted with lubricants containing only the proteins. Time-dependent frictional behaviors for all 3 lubricants are shown in Fig. 2. $\gamma$-globulin seems to be more effective lubricant than albumin despite its lower concentration in solution. The initial values of friction coefficient are 0.04 for $\gamma$-globulin and 0.1 for albumin which is more than two times higher value. At the end of the measurement, friction coefficient reached the highest values for both solutions -0.14 for albumin and 0.28 for $\gamma$-globulin. $\gamma$-globulin has a strong hydrophobic nature (Murakami, 2011) and in aqueous solutions can adsorb quite easily onto friction surfaces. Moreover, it shows strong bonding with mating molecules. On the other hand, albumin with superior hydrophilic property creates layers with low shearing resistance and thus can easily desorb.

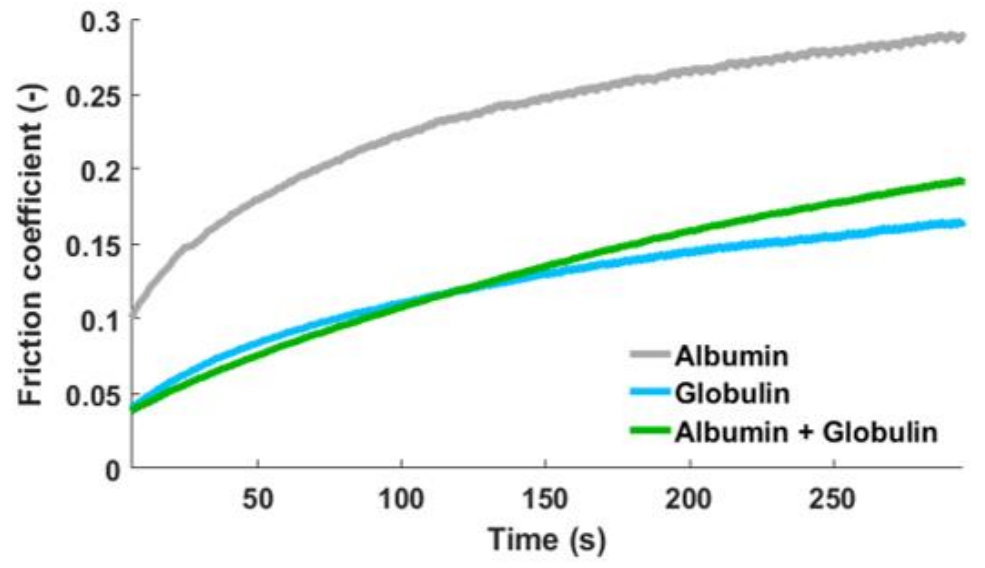

Fig. 2: Frictional behavior of protein solutions without hyaluronic acid.

Values of friction coefficient for solution containing both proteins are very similar with $\gamma$-globulin in first two minutes of the test. After this, they start to increase more rapidly. This can be explained by mechanism published by Nečas (2015). During adsorption of proteins on solid surfaces, $\gamma$-globulin forms thin protein layer; therefore, friction is very similar to $\gamma$-globulin solution at the beginning of the test. After this, albumin adsorbs onto this layer and friction coefficient starts to increase more rapidly.

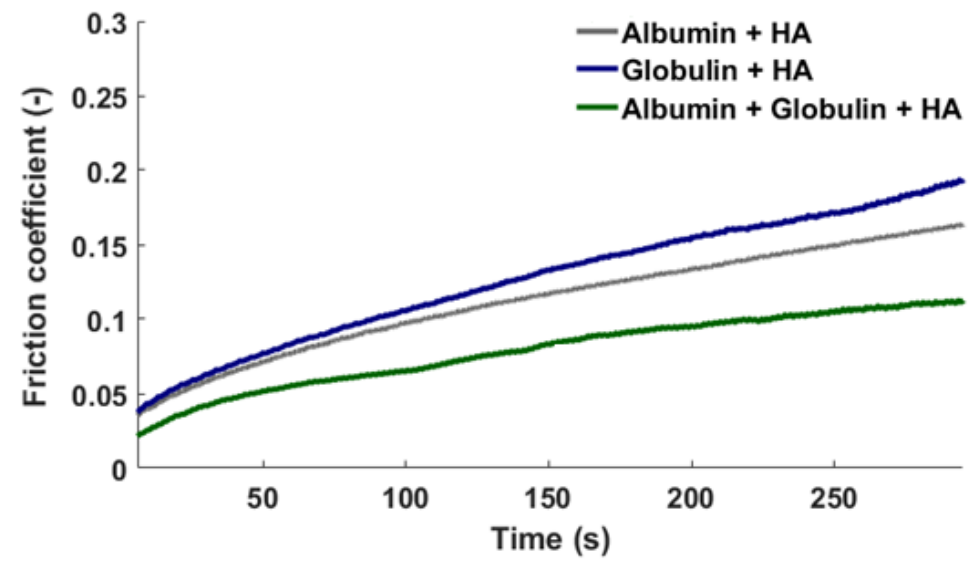

Fig. 3: Frictional behavior of protein solutions with hyaluronic acid. 
Result for solutions containing hyaluronic acid are shown in Fig. 3. The highest values of friction coefficient are obtained for solution of $\gamma$-globulin and hyaluronic acid. Friction is even higher than in the case of simple $\gamma$-globulin solution. $\gamma$-globulin probably prevents adsorption of hyaluronic acid onto cartilage surface, leading to increase of friction eventually. Mixing of albumin and hyaluronic acid leads to decrease of friction. Friction coefficient dropped from 0.28 to 0.15 after 300 seconds of the test. Mixing of these two substances seems to be synergistic. This effect is not so significant in the case of solution of all three substances due to presence of $\gamma$-globulin in solution.

\section{Conclusion}

In this study, changes of friction as a function of time for intact articular cartilage against glass plate were observed. Contact was lubricated with solutions containing proteins albumin and $\gamma$-globulin and hyaluronic acid. $\gamma$-globulin seems to be more effective lubricant than albumin due to stronger adsorption on cartilage surface. Mixture of both proteins exhibits the lowest initial values of friction coefficient. The coexistence of $\gamma$-globulin and hyaluronic acid was not effective for reduction of friction. On the other side, coexistence of hyaluronic acid with albumin or with mixture of proteins seems to be synergistic. This effect is more noticeable in the case of albumin solution. For detailed clarification of presented data could be helpful in situ observation of contact through optical methods. Their benefit was already presented in the field of biotribology of hip joint replacements (Nečas, 2015 and Nečas, 2018).

\section{Acknowledgement}

This research was carried out under the project LTAUSA17150 with financial support from the Ministry of Education, Youth and Sports of the Czech Republic. The authors would also like to thank the students from the fourth year for their lasting design collaboration.

\section{References}

Altman, R. D. et al. (2015) The mechanism of action for hyaluronic acid treatment in the osteoarthritic knee: a systematic review. BMC musculoskeletal disorders, 16, 1, pp. 321-330.

Balazs, E. A. and Denlinger, J. L. (1993) Viscosupplementation: a new concept in the treatment of osteoarthritis. The Journal of rheumatology. Supplement, 39, pp. 3-9.

Bell, C. J., Ingham, E. and Fisher, J. (2006) Influence of hyaluronic acid on the time-dependent friction response of articular cartilage under different conditions. Proceedings of the Institution of Mechanical Engineers, Part H: Journal of Engineering in Medicine, 220, 1, pp. 23-31.

Forsey, R. W. et al. (2006) The effect of hyaluronic acid and phospholipid based lubricants on friction within a human cartilage damage model. Biomaterials, 27, 26, pp. 4581-4590.

Galandáková, A. et al. (2017) Characteristics of synovial fluid required for optimization of lubrication fluid for biotribological experiments. Journal of Biomedical Materials Research Part B: Applied Biomaterials, 105, 6, pp. 1422-1431.

Ghosh, P. and Guidolin, D. (2002) Potential mechanism of action of intra-articular hyaluronan therapy in osteoarthritis: are the effects molecular weight dependent?. Seminars in arthritis and rheumatism, 32, 1, pp. 10-37.

Lees, D. and Partington, P. (2016) Articular cartilage. Orthopaedics and Trauma, 30, 3, pp. 265-272.

Murakami, T. et al. (2007) Micro-and nanoscopic biotribological behaviours in natural synovial joints and artificial joints. Proceedings of the Institution of Mechanical Engineers, Part J: Journal of Engineering Tribology, 221, 3 pp. 237-245.

Murakami, T. et al. (2011) Effectiveness of adsorbed film and gel layer in hydration lubrication as adaptive multimode mechanism for articular cartilage. Proceedings of the Institution of Mechanical Engineers, Part J: Journal of Engineering Tribology, 225, 12, pp. 1174-1185

Murakami, T. et al. (2013) Influence of synovia constituents on tribological behaviors of articular cartilage. Friction, 1,2 , pp. 150-162.

Nečas, D. et al. (2015) The effect of lubricant constituents on lubrication mechanisms in hip joint replacements. Journal of the mechanical behavior of biomedical materials, 55, pp. 295-307.

Nečas, D. et al. (2018) In situ observation of lubricant film formation in THR considering real conformity: The effect of model synovial fluid composition. Tribology International, 117, 1, pp. 206-216.

Schmidt, T. A. et al. (2007) Boundary lubrication of articular cartilage: role of synovial fluid constituents. Arthritis \& Rheumatology, 53, 3, pp. 882-891

Szarko, M., Muldrew, K. and Bertram, J. E. (2010) Freeze-thaw treatment effects on the dynamic mechanical properties of articular cartilage. BMC musculoskeletal disorders, 11, 1, pp. 231-238. 